\title{
Recent results and highlights from the ATLAS experiment
}

\author{
D. Caforio
}

Institute of Experimental and Applied Physics, CTU in Prague

on behalf of the ATLAS collaboration

Diffraction 2016, 03/09/2016 


\section{Overview}

(1) The ATLAS detector

- Overall data-taking efficiency and detector status

- Detector performance

(2) Overview of the most recent results

- $\mathrm{W}$ and $\mathrm{Z}$ production at $13 \mathrm{TeV}$

- Di-boson production

- Top quark

- Higgs

- Summary of SM results

- BSM results

- Diphoton resonances

- Supersymmetry

- Dark matter

(3) Summary and conclusions 


\section{ATLAS at the LHC}

The ATLAS collaboration

- 180 institutions

- 38 countries
- 3000 physicists
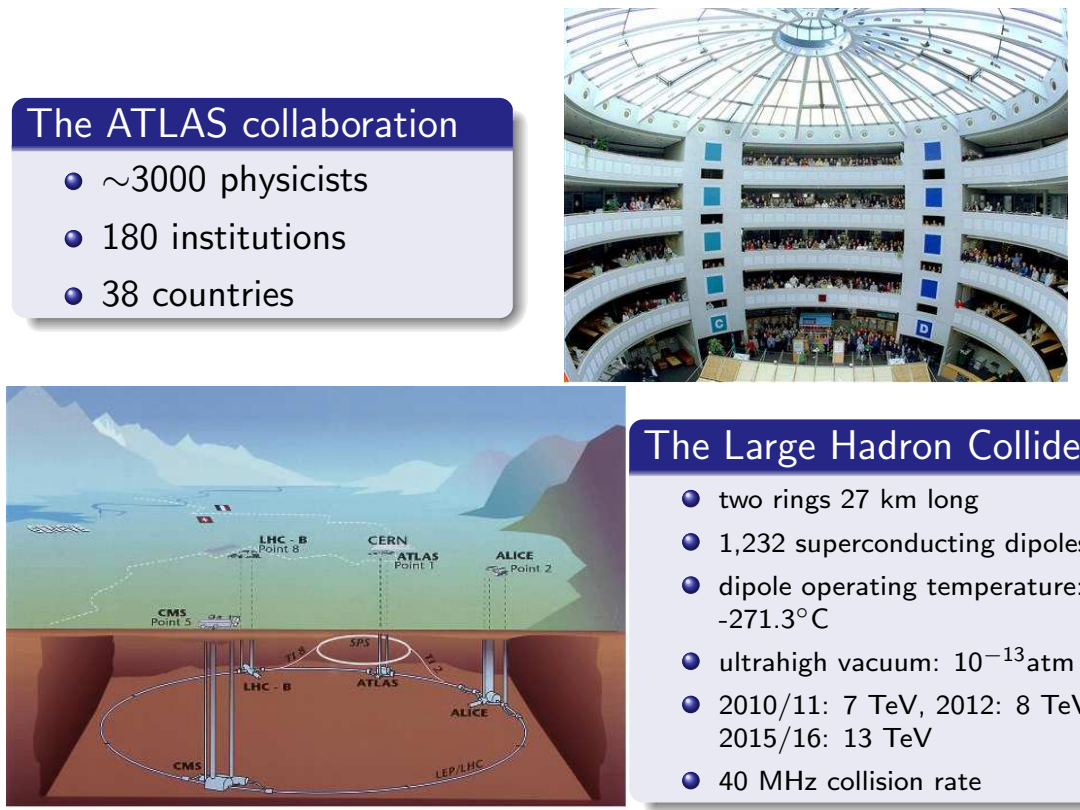

The Large Hadron Collider

- two rings $27 \mathrm{~km}$ long

- 1,232 superconducting dipoles

- dipole operating temperature: $-271.3^{\circ} \mathrm{C}$

- ultrahigh vacuum: $10^{-13} \mathrm{~atm}$

- 2010/11: 7 TeV, 2012: 8 TeV, 2015/16: $13 \mathrm{TeV}$

- $40 \mathrm{MHz}$ collision rate 


\section{The ATLAS detector}

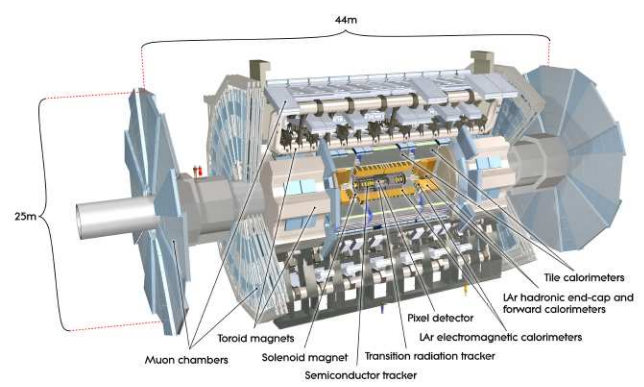

\section{The ATLAS detector}

- Inner Detector: Pixel, SCT, TRT

- Calorimeters: LAr (EM + hadronic + forward), TileCal (hadronic)

- Muon Spectrometers: barrel and endcaps

- Forward detectors: AFP, ALFA, LUCID, ZDC

- 2 magnetic fields: solenoidal (ID) and toroidal (MS)

- $150 \cdot 10^{6}$ read-out channels

\section{The ATLAS trigger system}

- Three levels: L1 is fully hardware, implemented in the calorimeters and the Muon Spectrometer

- L2 and the Event Filter (EF) are software based: the L2 accepts data from defined Regions Of Interests (ROI) of L1

- EF provides a full event reconstruction on computer farms

- $10^{14} \mathrm{~B} / \mathrm{s}$ raw data flux

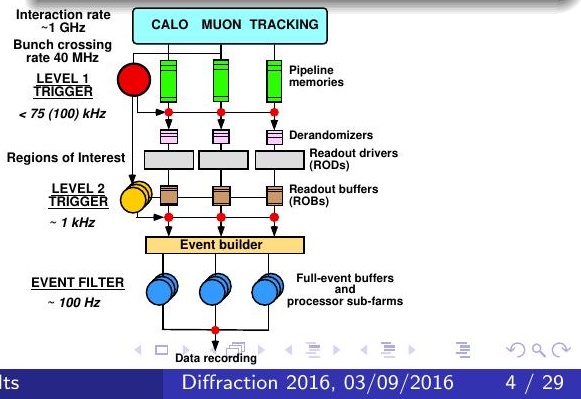




\section{Overall data-taking efficiency and detector status}

- $3.9 \mathrm{fb}-1 \mathrm{pp}$ at $13 \mathrm{TeV}$ recorded in 2015 (92\% DAQ efficiency)

- $19.3 \mathrm{fb}-1 \mathrm{pp}$ at $13 \mathrm{TeV}$ recorded in 2016 (as of August $15^{\text {th }}$ )

- peak luminosity delivered by LHC: $1.15 \cdot 10^{34} \mathrm{~cm}^{-2} \mathrm{~s}^{-1}$ (greater than design value 1 . $\left.10^{34} \mathrm{~cm}^{-2} \mathrm{~s}^{-1}\right)$
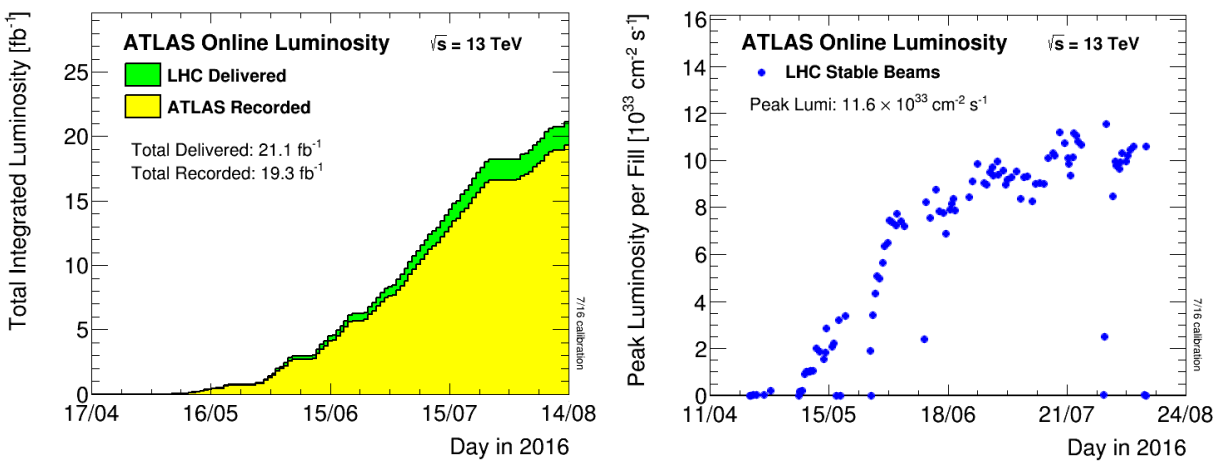


\section{Overall data-taking efficiency and detector status}

\section{ATLAS pp 25ns run: April-July 2016}

\begin{tabular}{|ccccccccccccc|}
\multicolumn{4}{c}{ Inner Tracker } & \multicolumn{3}{c}{ Calorimeters } & \multicolumn{3}{c|}{ Muon Spectrometer } & \multicolumn{2}{c|}{ Magnets } \\
\hline Pixel & SCT & TRT & LAr & Tile & MDT & RPC & CSC & TGC & Solenoid & Toroid \\
\hline 98.9 & 99.9 & 100 & 99.8 & 100 & 99.6 & 99.8 & 99.8 & 99.8 & 99.7 & 93.5 \\
\hline
\end{tabular}

\section{Good for physics: $91-98 \%\left(10.1-10.7 \mathrm{fb}^{-1}\right)$}

Luminosity weighted relative detector uptime and good data quality efficiencies (in \%) during stable beam in $\mathrm{pp}$ collisions with $25 \mathrm{~ns}$ bunch spacing at $\sqrt{s}=13 \mathrm{TeV}$ between 28th April and 10th July 2016, corresponding to an integrated luminosity of $11.0 \mathrm{fb}^{-1}$. The toroid magnet was off for some runs, leading to a loss of $0.7 \mathrm{fb}^{-1}$. Analyses that don't require the toroid magnet can use that data.

- fraction of operational channels $>96 \%$

- $\mathrm{DQ}$ losses $<1 \%$ for each individual system 


\section{Detector performance}

Momentum scale and resolution of the Muon Spectrometer studied in detail using $J / \psi \rightarrow \mu \mu$ and $Z \rightarrow \mu \mu$ decays

Jet energy scale uncertainty at the level of 2012 data
Eur. Phys. J. C 76 (2016): 292
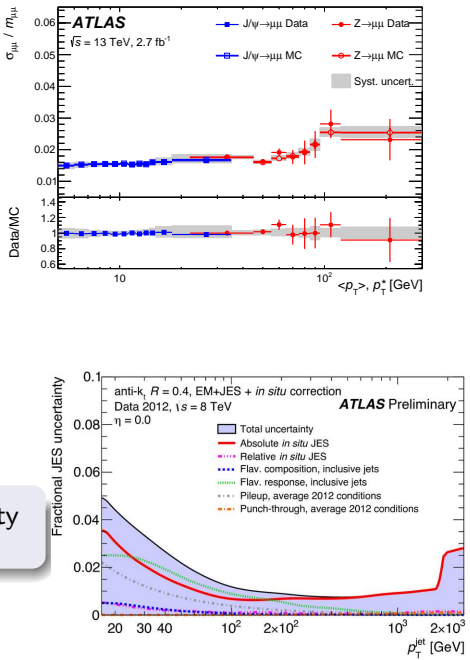
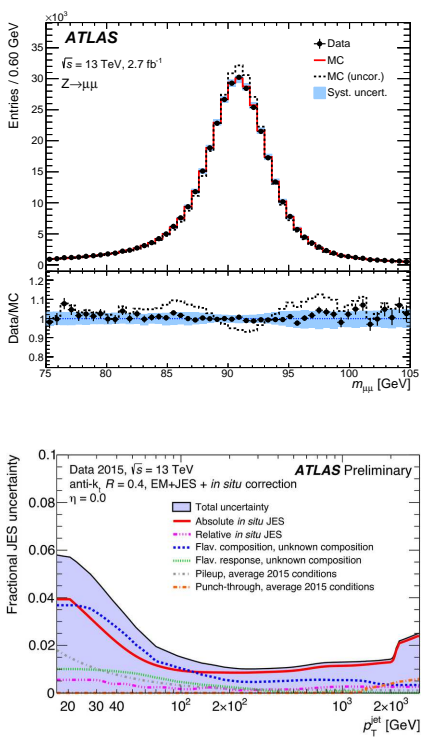


\section{$\mathrm{W}$ and $\mathrm{Z}$ production at $13 \mathrm{TeV}$}

- both absolute cross sections and ratios measured

- measurements of cross-section ratios benefit from the cancellation of some experimental uncertainties, and are powerful tools to constrain PDF fits

- $\frac{W^{+}}{W^{-}}$: sensitive to $\mathrm{u}$ and $\mathrm{d}$ quark valence distribution, measured to a precision of $0.8 \%$

- $\frac{W^{+/-}}{Z}$ : sensitive to strange quark content
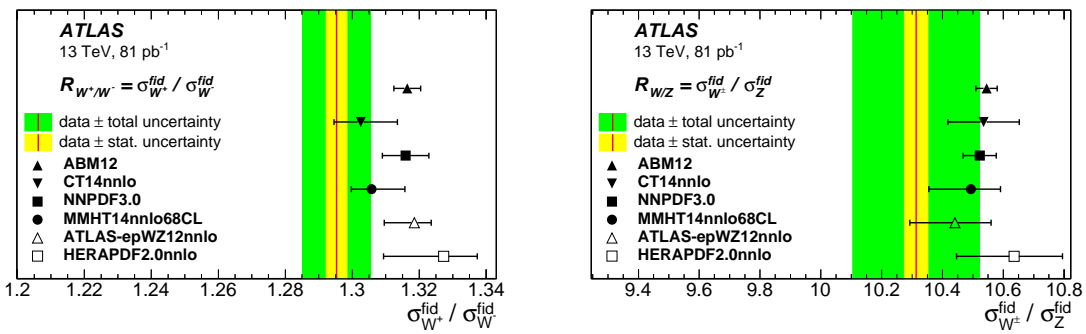

Phys. Lett. B 759 (2016) 601 


\section{Di-boson production}

- test of the SM at TeV scale

- important for background estimations needed for many measurements

- new physics could manifest in $W^{ \pm} Z$ final states as a modification of the TGC and QGC strength

- precise knowledge of the $W^{ \pm} Z$ production cross section is necessary in the search for new physics

- only a short selection shown here: more results available 


\section{Di-boson production: $\mathrm{WZ}$}

- Run-1 results were above the NLO predictions

- Run-2 total cross-section found to be consistent with very recent NNLO calculations
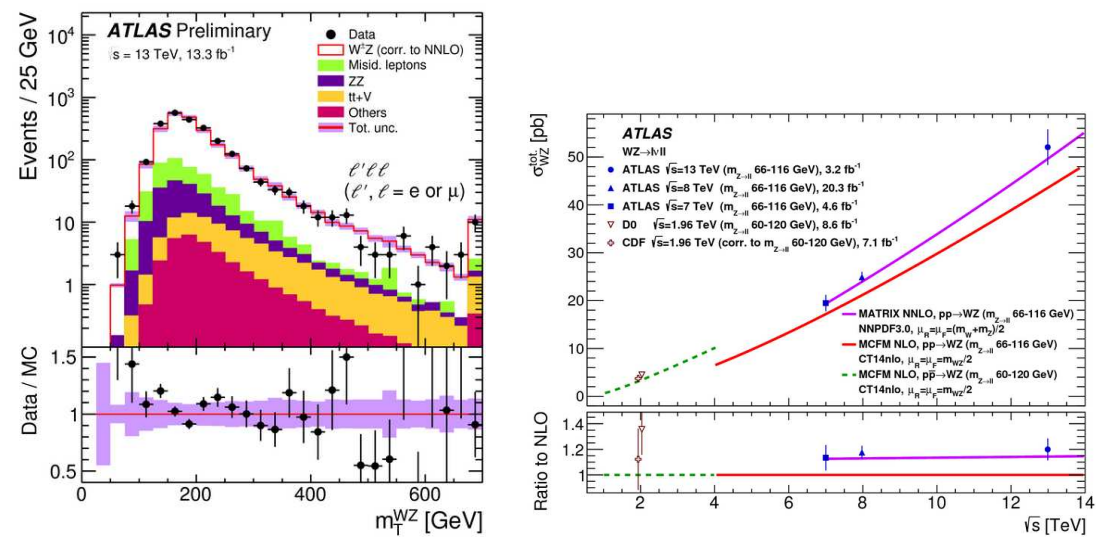

arXiv: 1606.04017 


\section{Di-boson production: WW}

- cross-section measurement of $W^{+} W^{-} \rightarrow e^{ \pm} \nu \mu^{\mp} \nu$ production at $13 \mathrm{TeV}$

- agreement with NNLO prediction at $13 \mathrm{TeV}$
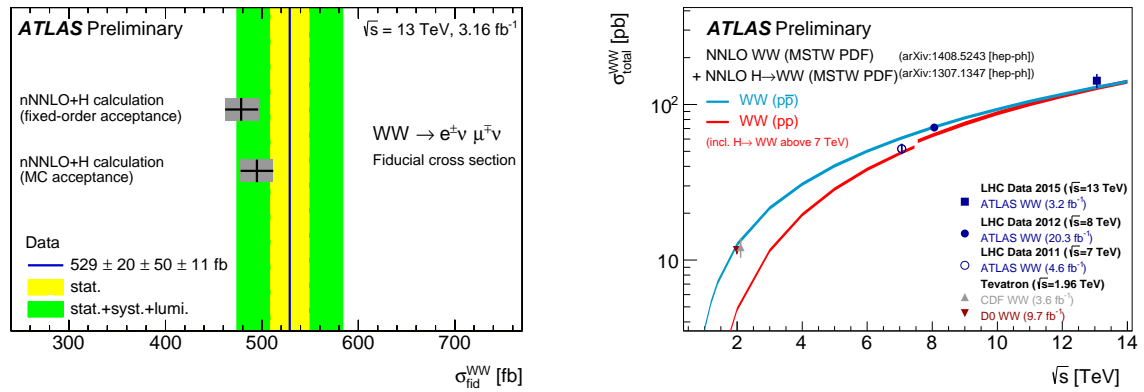

ATLAS-CONF-2016-090 


\section{Top quark}

- heaviest of the known fundamental particles

- at LHC, with luminosity $\sim 10^{34} \mathrm{~cm}^{-2} \mathrm{~s}^{-1}$, top pair production rate is $\sim 8$ pairs $/ \mathrm{s}$

- it decays before hadronisation: its properties are transferred directly to its decay products (information on "bare quarks")

- the Standard Model predicts all its properties given the mass

- deviations may point to "new physics"

- $\mathrm{ttH}$ coupling studied also in ttH production: precision measurements of Higgs boson coupling to top quarks can reveal BSM effects 


\section{Top pair production cross-section}

- inclusive top pair production in good agreement with NNLO prediction

- experimental measurements have reached the theoretical calculation

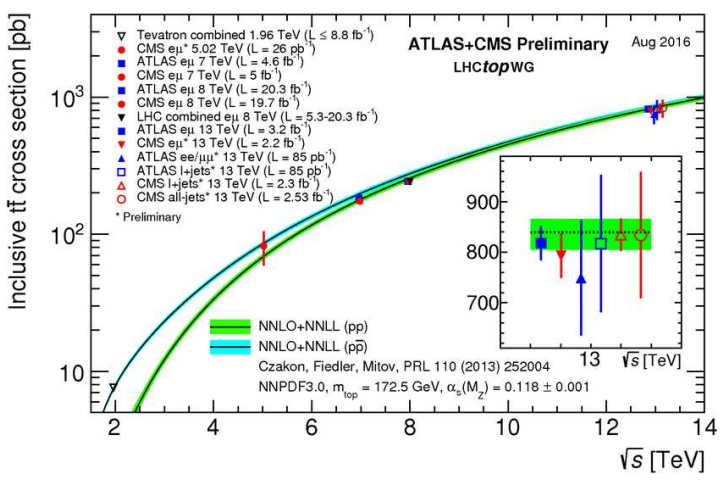

$\sigma_{t \bar{t}}=818 \pm 8$ (stat) \pm 27 (syst) \pm 19 (lumi) \pm 12 (beam) pb at $\sqrt{s}=13 \mathrm{TeV}$ 


\section{Top quark mass}

Measured both directly from invariant mass and derived from cross-section (pole mass determination)

Recent direct measurement

from $\mathrm{tt} \rightarrow$ dilepton at $8 \mathrm{TeV}$

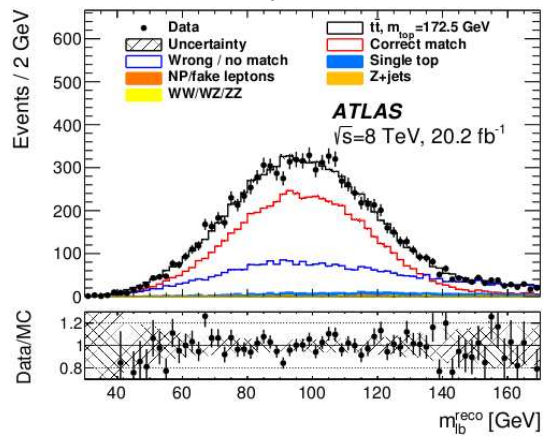

Pole mass determination

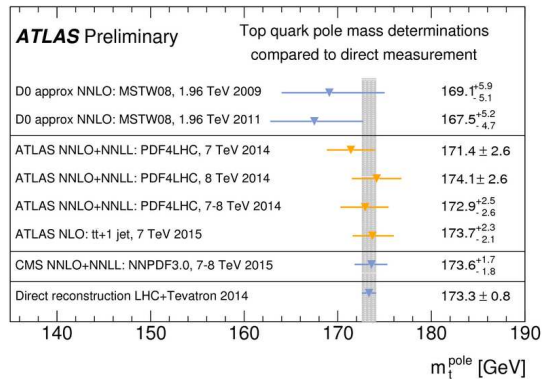

$$
m_{\text {top }}=172.84 \pm 0.34 \text { (stat) } \pm 0.61 \text { (syst) } \mathrm{GeV}=172.84 \pm 0.70 \mathrm{GeV}
$$

arXiv: 1606.02179 


\section{Top couplings with $\mathrm{Z}$ and $\mathrm{W}$}

- associated production of $t \bar{t}$ with a $\mathrm{Z}$ boson allow to extract information on the neutral current coupling of the top quark

- production rate of a top-quark pair with a massive vector boson could be altered in the presence of physics beyond the Standard Model (vector-like quarks, strongly coupled Higgs bosons or technicolor)

- at $13 \mathrm{TeV}$ the SM cross sections of the ttZ and ttW processes increase by factors of 3.5 and 2.4 , respectively, compared to $8 \mathrm{TeV}$
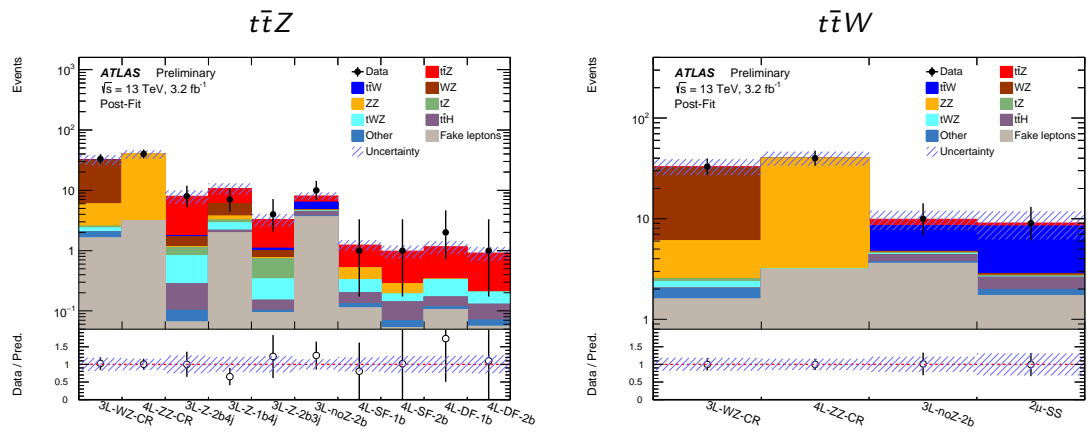

both measurements consistent with the NLO QCD theoretical calculations ATLAS-CONF-2016-003 


\section{Higgs}

- 2015+2016 data

- fiducial and differential cross section measurements

- event categories enhancesensitivity to measurement of production mode cross sections: sigma_ggH, sigma_VBF, ...

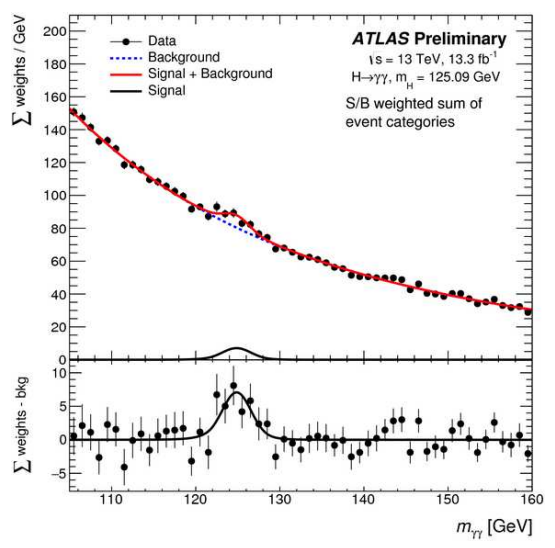

ATLAS-CONF-2016-067

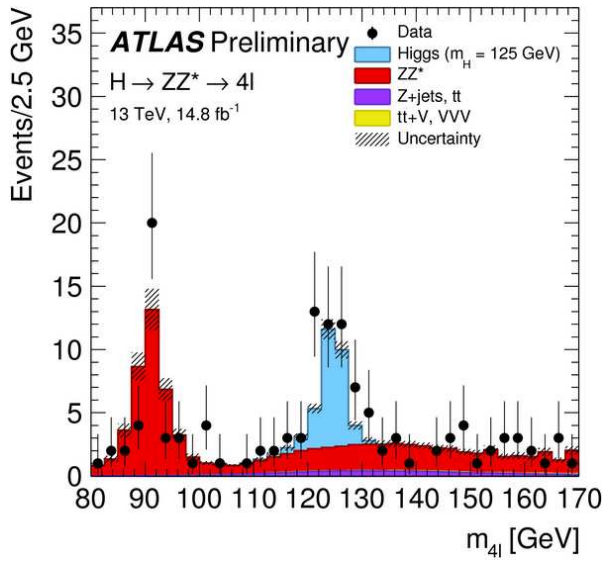

ATLAS-CONF-2016-079 


\section{Higgs}

Combining $\gamma \gamma+4 /$ channels (combination of fiducial cross sections):

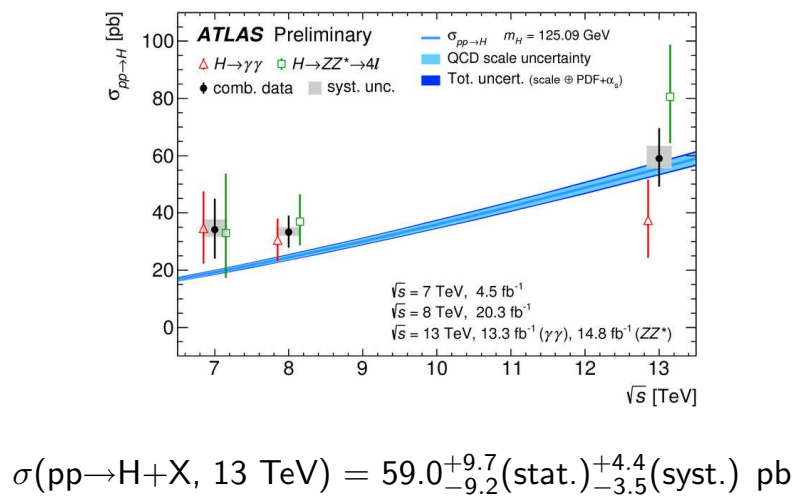

- SM prediction: $55.5_{-3.4}^{+2.4} \mathrm{pb}$

- overall significance at $13 \mathrm{TeV}: \sim 10 \sigma$

ATLAS-CONF-2016-081 


\section{ttH production}

- direct probe of top Yukawa coupling

- cross-section at $13 \mathrm{TeV} 4$ times larger than at $8 \mathrm{TeV}$

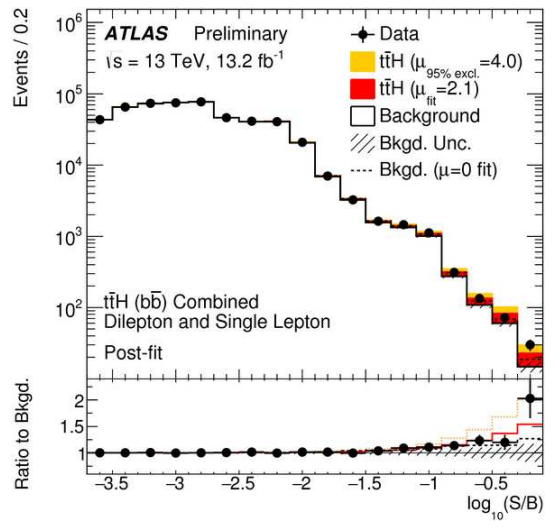

ATLAS-CONF-2016-080

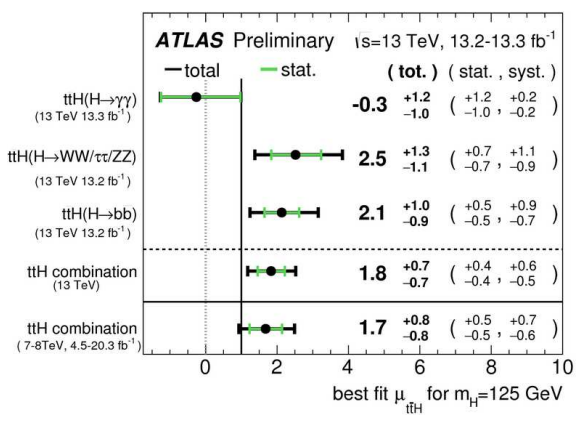

ATLAS-CONF-2016-068 


\section{Summary of selected SM results}

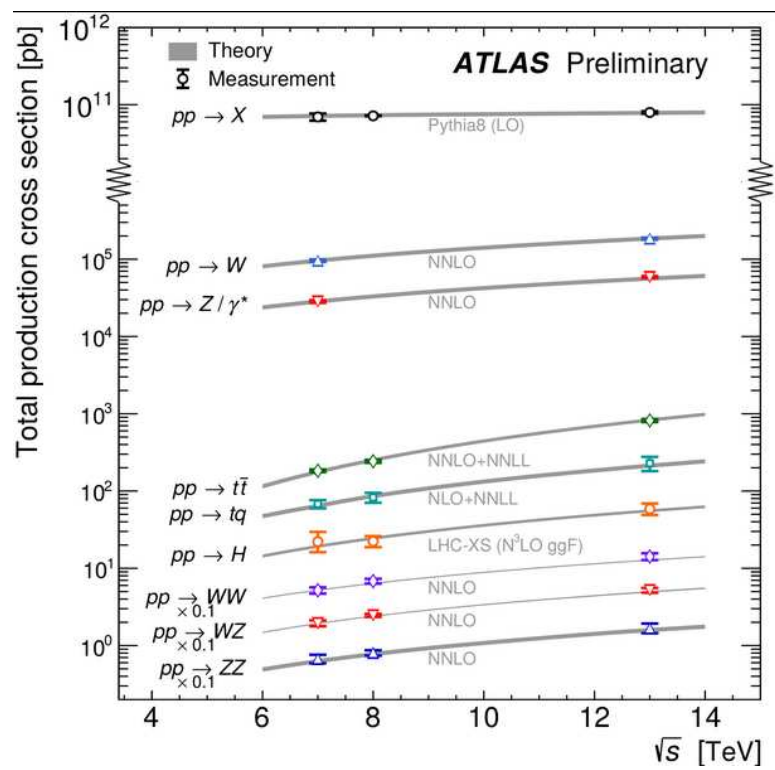

오 $p p \rightarrow X$

$7 \mathrm{TeV}, 20 \mu \mathrm{b}^{-1}$, Nat. Commun. 2, 463 (2011)

$8 \mathrm{TeV}, 500 \mu \mathrm{b}^{-1}$, arXiv: 1607.06605

$13 \mathrm{TeV}, 60 \mu \mathrm{b}^{-1}$, arXiv: 1606.02625

工्s $p p \rightarrow W \quad \overline{7} p p \rightarrow Z / \gamma^{*}$

$7 \mathrm{TeV}, 36 \mathrm{pb}^{-1}$, PRD 85, 072004 (2012)

$13 \mathrm{TeV}, 81 \mathrm{pb}^{-1}$, PLB 759 (2016) 601

$\bar{\Sigma} p p \rightarrow t \bar{t}$

$7 \mathrm{TeV}, 4.6 \mathrm{fb}^{-1}$, Eur. Phys. J. C 74:3109 (2014)

8 TeV, $20.3 \mathrm{fb}^{-1}$, Eur. Phys. J. C 74:3109 (2014)

$13 \mathrm{TeV}, 3.2 \mathrm{fb}^{\mathrm{-1}}$, arXiv:1606.02699

도 $p p \rightarrow t q$

$7 \mathrm{TeV}, 4.6 \mathrm{fb}^{-1}$, PRD 90, 112006 (2014)

$8 \mathrm{TeV}, 20.3 \mathrm{fb}^{-1}$, ATLAS-CONF-2014-007

$13 \mathrm{TeV}, 3.2 \mathrm{fb}^{-1}$, ATLAS-CONF-2015-079

ठ $p p \rightarrow H$

$7 \mathrm{TeV}, 4.5 \mathrm{fb}^{-1}$, Eur. Phys. J. C76 (2016) 6

$8 \mathrm{TeV}, 20.3 \mathrm{fb}^{-1}$, Eur. Phys. J. C76 (2016) 6

$13 \mathrm{TeV}, 13.3 \mathrm{fb}^{-1}$, ATLAS-CONF-2016-081

\. $p p \rightarrow W W$

$7 \mathrm{TeV}, 4.6 \mathrm{fb}^{-1}$, PRD 87, 112001 (2013)

$8 \mathrm{TeV}, 20.3 \mathrm{fb}^{-1}$, arXiv: 1608.03086

$13 \mathrm{TeV}, 3.2 \mathrm{fb}^{-1}$, ATLAS-CONF-2016-090

$\overline{7} p p \rightarrow W Z$

$7 \mathrm{TeV}, 4.6 \mathrm{fb}^{-1}$, Eur. Phys. J. C (2012) 72:2173

$8 \mathrm{TeV}, 20.3 \mathrm{fb}^{-1}$, PRD 93, 092004 (2016)

$13 \mathrm{TeV}, 3.2 \mathrm{fb}^{-1}$, arXiv: 1606.04017

T $p p \rightarrow Z Z$

$7 \mathrm{TeV}, 4.6 \mathrm{fb}^{-1}$, JHEP 03, 128 (2013)

$8 \mathrm{TeV}, 20.3 \mathrm{fb}^{-1}$, ATLAS-CONF-2013-020

$13 \mathrm{TeV}, 3.2 \mathrm{fb}^{-1}$, PRL 116, 101801 (2016) 


\section{Selected summary of Exotics results}

only a representative selection of the available results is shown

ATLAS Exotics Searches* - 95\% CL Exclusion

ATLAS Preliminary

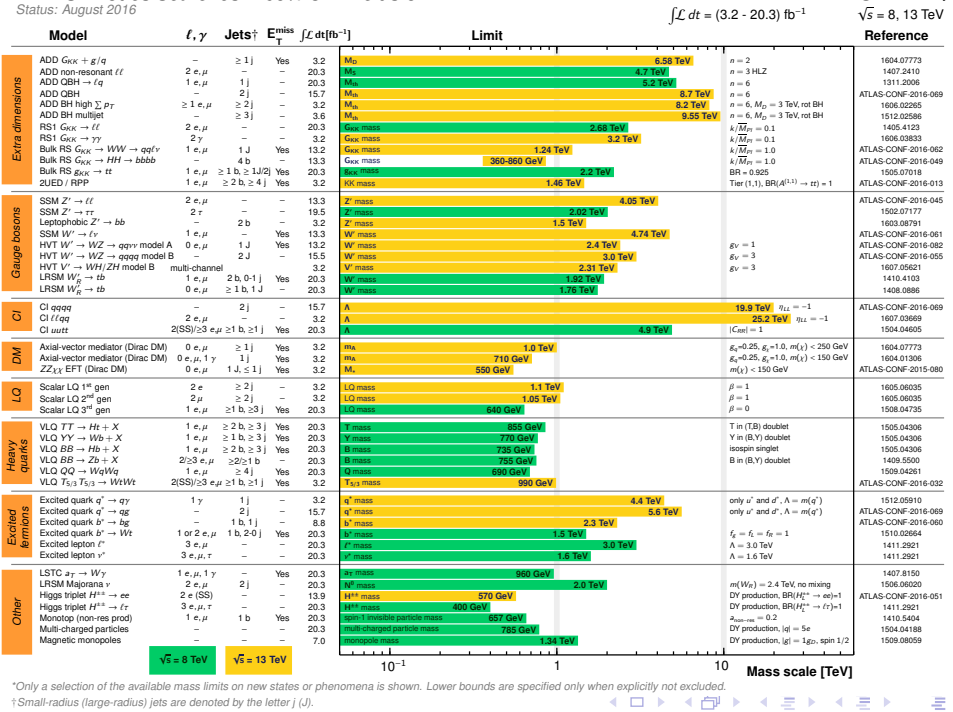




\section{$\mathrm{H} / \mathrm{A} \rightarrow \tau \tau$}

Some MSSM models predict high mass scalar decaying in high mass $\tau \tau$ :

- two decay modes: lepton + hadron and hadron-hadron

- main backgrounds: $\mathrm{Z} \tau \tau$ and multijets
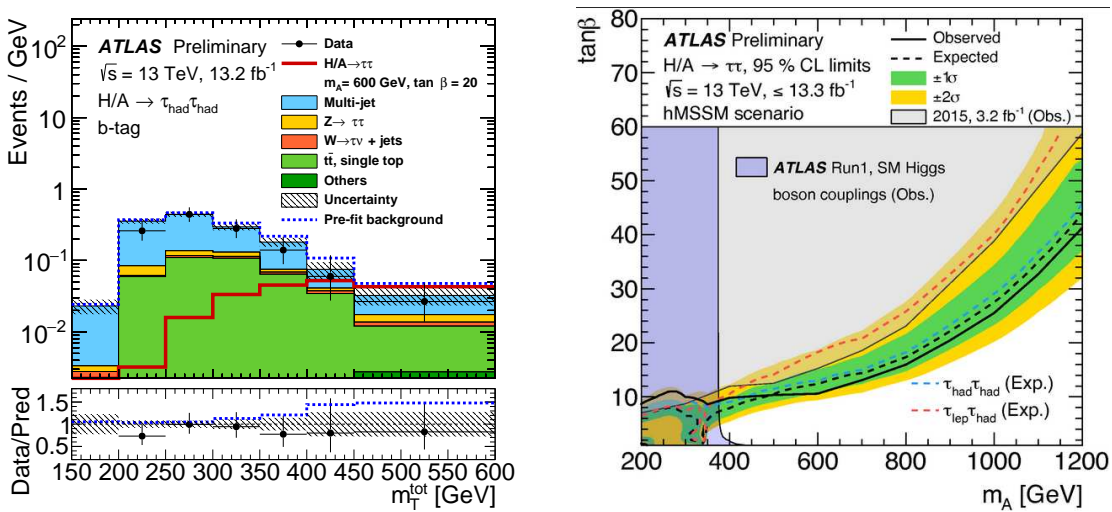


\section{Diphoton resonances}

\section{5 data:}

- excess at $750 \mathrm{GeV}$ (after reprocessing, at $730 \mathrm{GeV}$ )

- local significance $3.9 \sigma$, global $2.1 \sigma$ (after reprocessing: local significance $3.4 \sigma)$

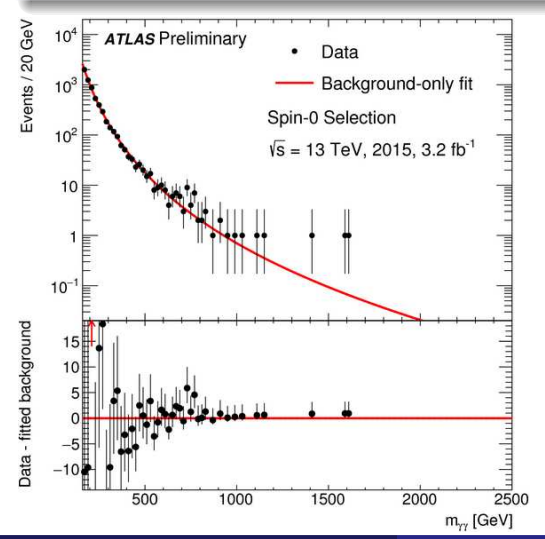

D. Caforio (IEAP - CTU, Prague)

\section{5+2016 data:}

- small excess at $710 \mathrm{GeV}$

- local significance $1.4 \sigma$, global $<1 \sigma$

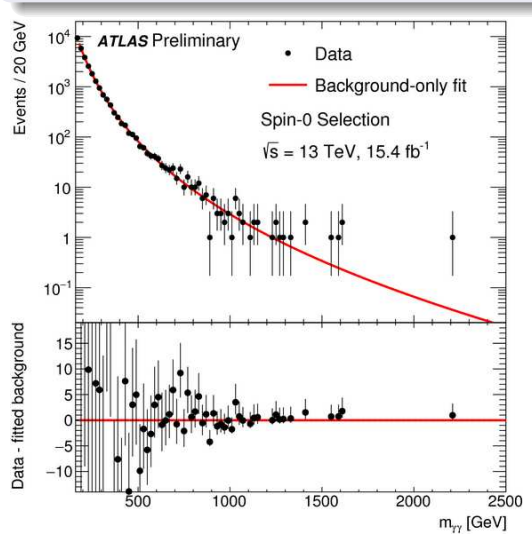




\section{Selected summary of Supersymmetry results}

only a representative selection of the available results is shown

ATLAS SUSY Searches* - 95\% CL Lower Limits

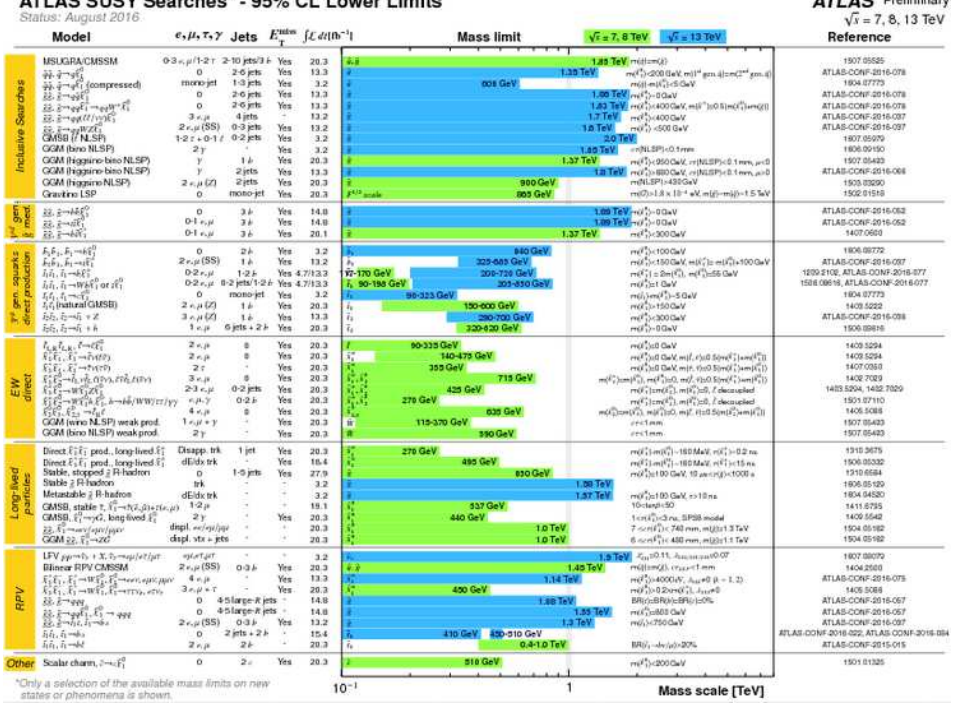




\section{Jets $+\mathrm{E}_{T}^{\text {miss }}(0 \ell)$}

- expect large increase of SUSY cross-sections going from $8 \rightarrow 13$ $\mathrm{TeV}: \sigma(\tilde{g} \tilde{g}) \times 30$ for $m(\tilde{g})=1.4 \mathrm{TeV}$

- signature: 0 lepton + jets (2 to 6$)+E_{T}^{\text {miss }}$

- main backgrounds: Z/W+jets and $t \bar{t}$

- example: $M_{\text {eff }}$ analysis
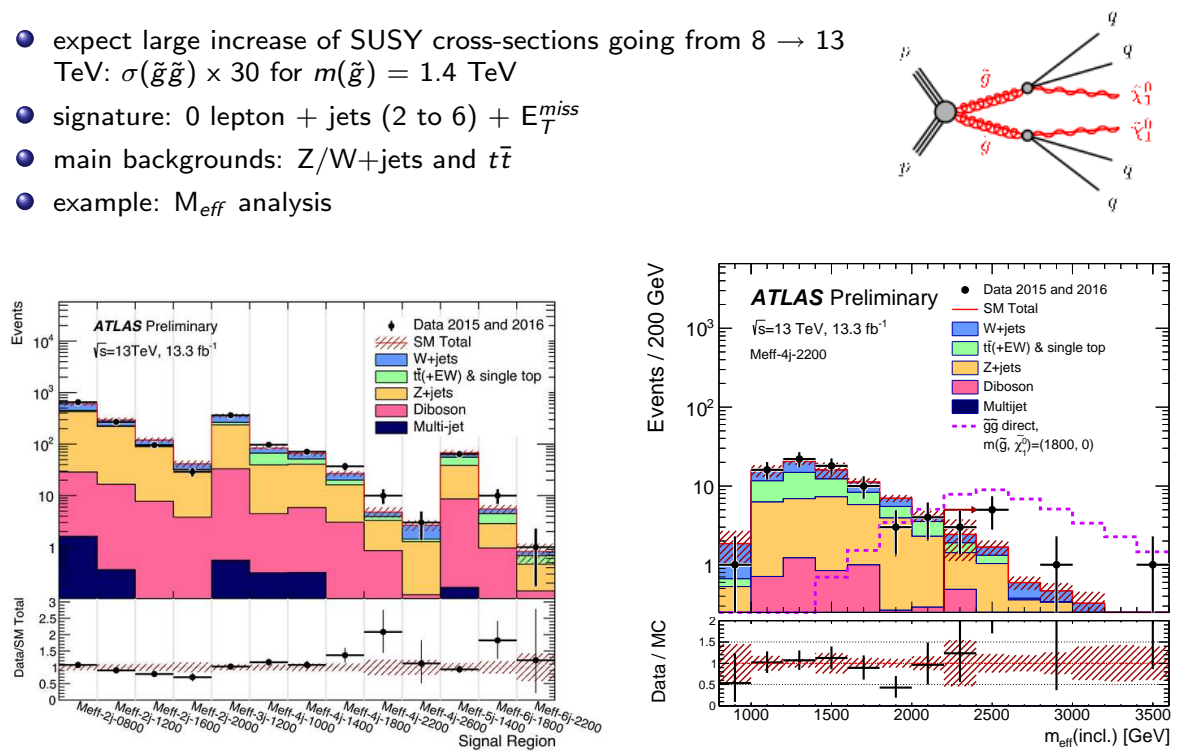


\section{Jets $+\mathrm{E}_{T}^{\text {miss }}(0 \ell)$}

- final limits in 0L final states with 2-6 jets

- no significant excesses

- new Recursive Jigsaw Reconstruction (RJR) technique implemented (not shown here)

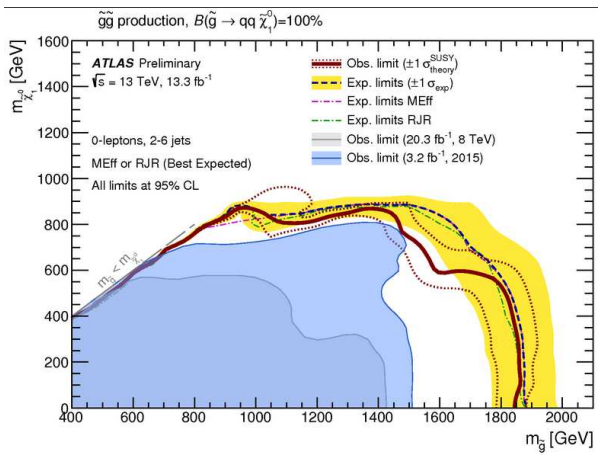

ATLAS-CONF-2016-078 


\section{stop}

- signature: 1 lepton + jets $+\mathrm{E}_{T}^{\text {miss }}$

- main backgrounds: Z/W+jets and $t \bar{t}$

- largest excess: $3.3 \sigma$ (local)
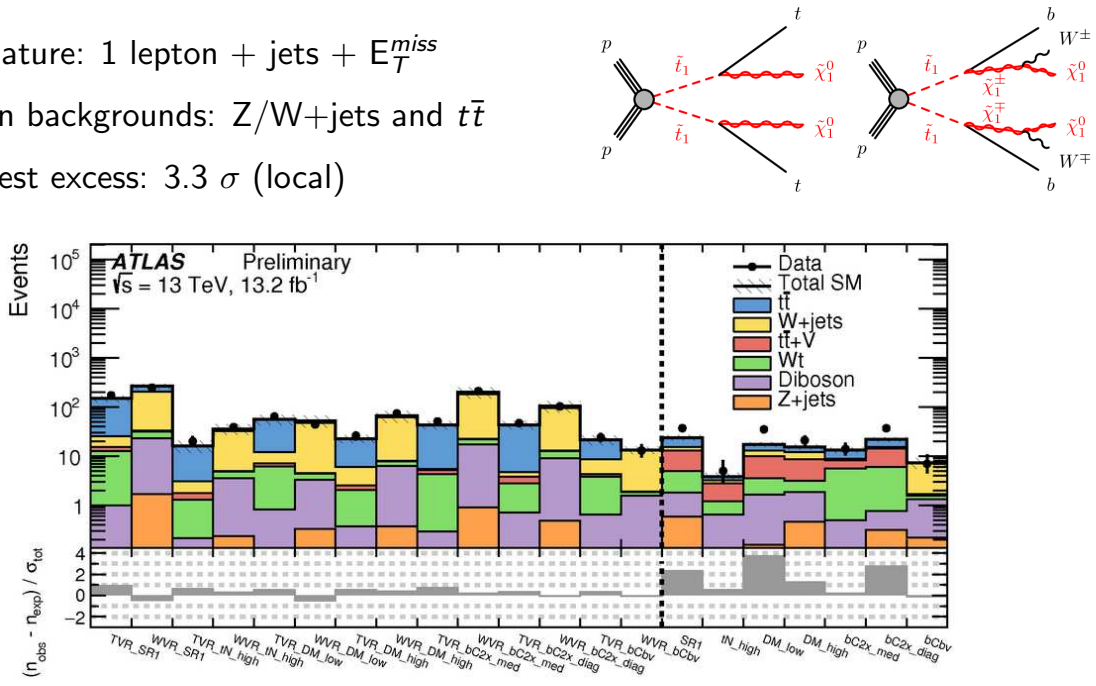

ATLAS-CONF-2016-050 


\section{stop}

summary of the dedicated ATLAS searches for top squark (stop) pair production based on $13 \mathrm{fb}-1$ of pp collision data taken at $\sqrt{s}=13 \mathrm{TeV}$

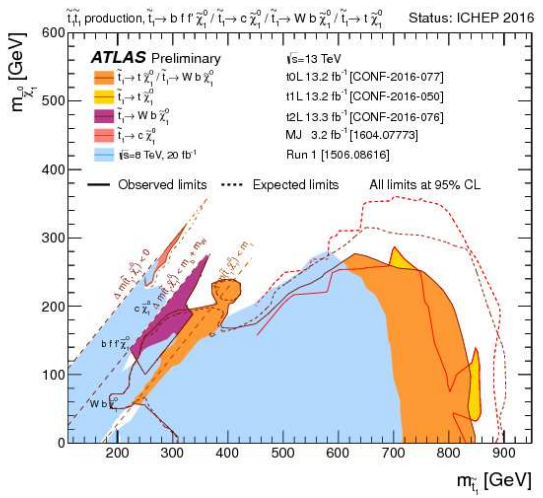

the yellow limit shows the result of the analysis discussed in the previous slide 


\section{Dark matter}

- searches for weakly-interacting dark matter production in association with other SM particles

- distinct signature of significant missing transverse momentum

- example: $\mathrm{E}_{T}^{\text {miss }}+\mathrm{ZZ}(\rightarrow \ell \ell)$

\section{ATLAS-CONF-2016-056}
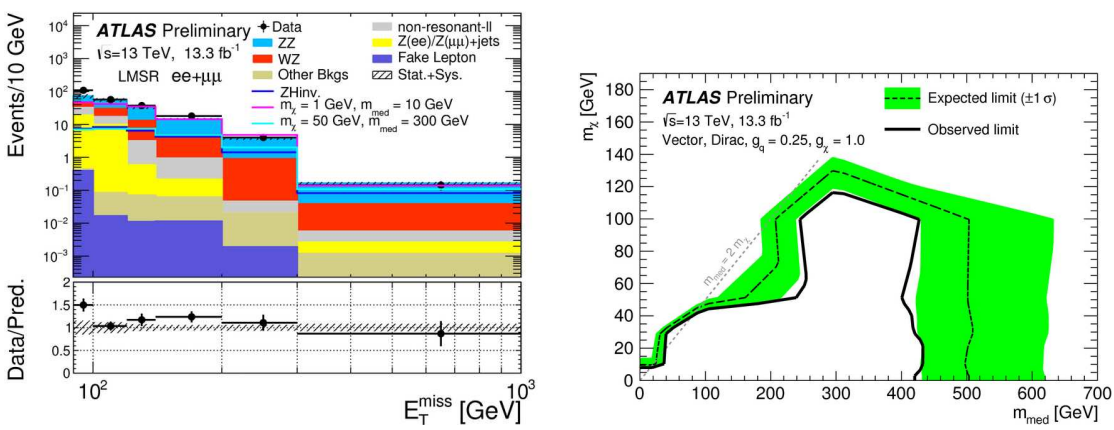

- no significant excesses observed

- limits have been set, search continues 


\section{Summary and conclusions}

- ATLAS is coping very well with LHC approaching (and overcoming) design luminosity

- many results already available:

- Higgs properties

- towards a ttH coupling measurement: observed significance of $2.8 \sigma$ (combined photon, multilepton, and b-quark decays)

- increased sensitivity to BSM searches

- almost 600 publications

- no significant excesses yet, more data coming soon

- expect more exciting Run-2 results within next 2 years: $100 \mathrm{fb}-1$ expected 